\section{A NOVEL ULTRA-WIDEBAND FREQUENCY PLANAR NOTCH-FILTER ANTENNA}

C. Gómez-Calero, ${ }^{1}$ B. TahaAhmed, ${ }^{2}$ and R. Martínez ${ }^{1}$ ${ }^{1}$ Radiation Group, Department of Signals, Systems and Radiocommunications, Universidad Politécnica de Madrid, Ciudad Universitaria, 28040 Madrid, Spain; Corresponding author: carlosgc@gr.ssr.upm.es

${ }^{2}$ Grupo de Sistemas de Radiocomunicaciones y Comunicaciones Ópticas (GSRCO), Universidad Autónoma de Madrid, Madrid, Spain

ABSTRACT: Many research works have emerged for ultra-wideband (UWB) systems. One important issue is the antenna design to cover all frequency bands $(3.1-10.6 \mathrm{GHz})$. In most applications, a reduced compact antenna is needed but with several requirements. In this paper, a novel triangle-semicircular planar monopole UWB antenna is presented with a notch filter to avoid the undesirable interferenses in ISM (Industrial, Scientific and Medical) band at $5.3 \mathrm{GHz}$

Key words: ultra-wideband; planar antenna; notch filter

\section{INTRODUCTION}

In the last decade, many new ultra-wideband (UWB) applications have appeared, and many prolific research works are emerging in this topic. UWB systems use ultra-short pulses in the order of nanoseconds to occupy a huge frequency band. Especially, USA Federal Communications Commission (US-

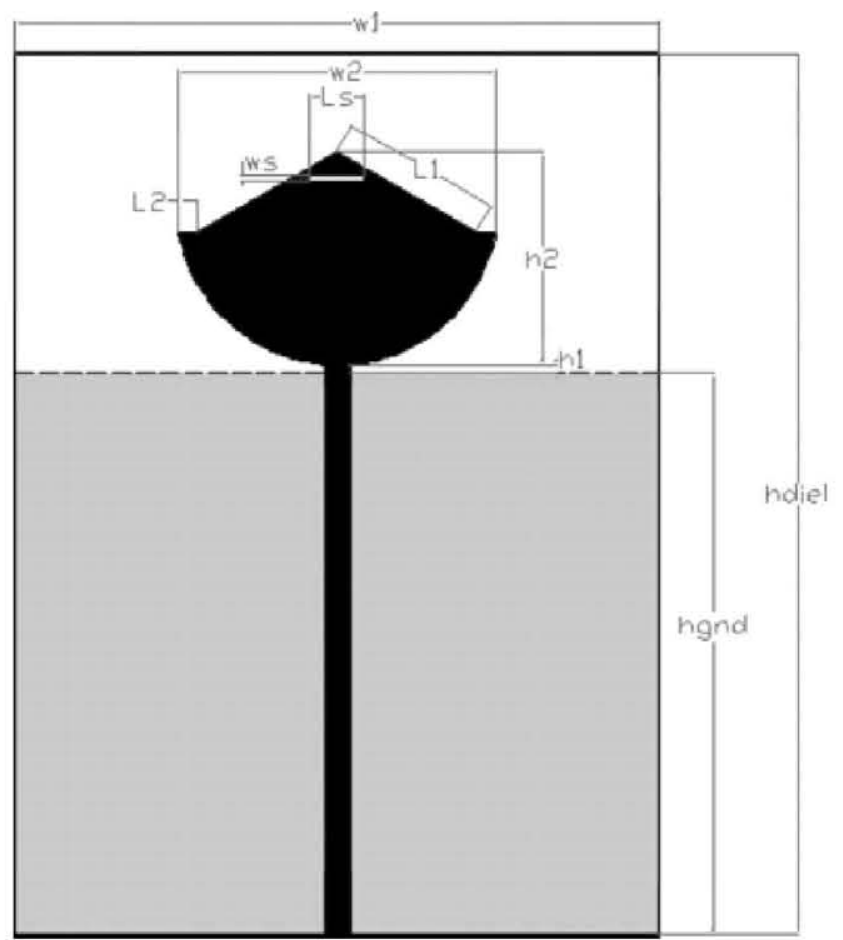

Figure 1 Top view and dimensions of UWB antenna. [Color figure can be viewed in the online issue, which is available at www.interscience.wiley.com]

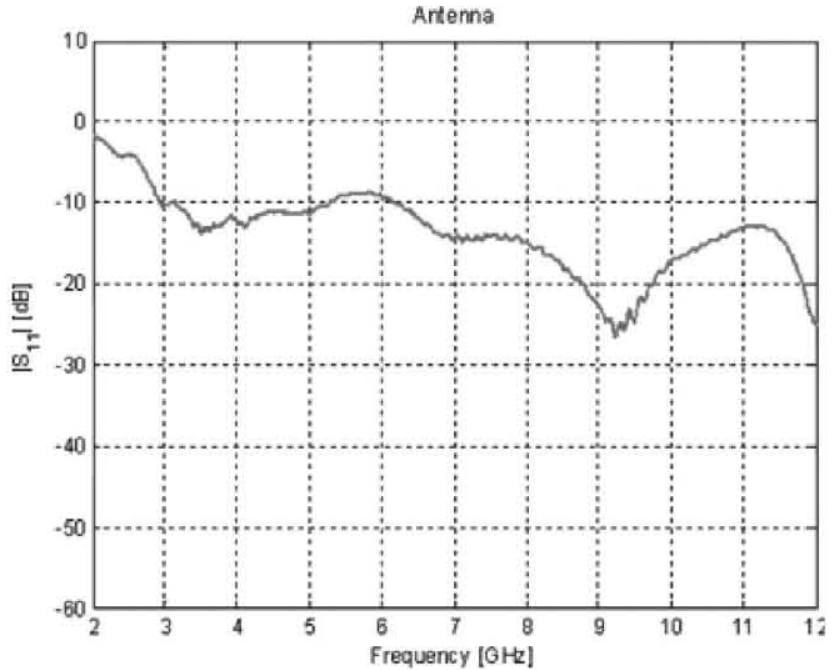

Figure 2 Reflection coefficient of the designed UWB antenna as function of the frequency range. [Color figure can be viewed in the online issue, which is available at www.interscience.wiley.com]

FCC) has assigned the frequency band of $3.1-10.6 \mathrm{GHz}$ with respect to these emerging UWB activities. The aim of UWB systems is to increase the data bit rate of different applications due to limitations in current wireless systems. Likewise, the UWB systems can achieve hundreds of Mbps, so many applications for these systems have appeared, such as microwave imaging because of the enhancement of resolution.

Regarding the radiating element for UWB, many antennas have been designed and presented [1]. The most commonly used are the printed antennas. Different shapes for UWB antennas have been devised, such as Vivaldi, bow-tie, or monopoles antennas. Even the use of frequency-independent antennas such as fractals has been emerged, but the performance of these antennas has generally been disappointing due to dispersion. Possibly, the monopole shape is the most commonly used antenna for UWB. Likewise, several planar monopole antennas with various shapes have been devised: square, circular, or elliptic, and in microstrip or coplanar waveguide technology [2, 3].

On the other hand, for a UWB antenna, the size and cost are important constraints. Printed antennas exemplified by the microstrip patch antenna offer an attractive solution to compact, conformal, and low-cost design of RF communication systems. In this paper, a low cost and easy to manufacture monopole antenna has been designed to cover the entire UWB frequency band by means of a microstrip structure. However, and due to the huge bandwidth, several systems such as wideband local area network (WLAN) create interferences in a certain range of frequencies. To avoid these interferences, several solutions for antenna designs have been proposed in literature [4]. Most of solutions try to vary the shape of the radiating element. Likewise, in this paper, a microstrip notch filter has been designed to separate the radiating element from the feeding network.

\section{ANTENNA DESIGN}

The radiating element has been designed to be a printed monopole matched overall the frequency bands (3.1-10.6 GHz). Thus, the antenna could be used in any UWB applications. The antenna structure is based on a microstrip design, where the dielectric is fiber glass with the dielectric constant $\varepsilon_{\mathrm{r}}=4.4$ and the height $0.9 \mathrm{~mm}$. At the bottom, the ground plane is situated and at the top of the structure, the feeding and the radiating 

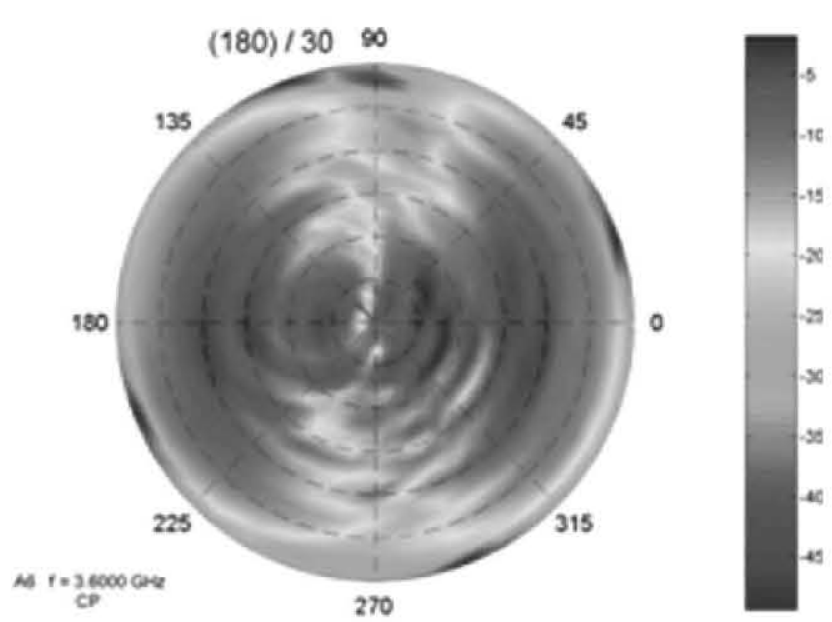

(a)

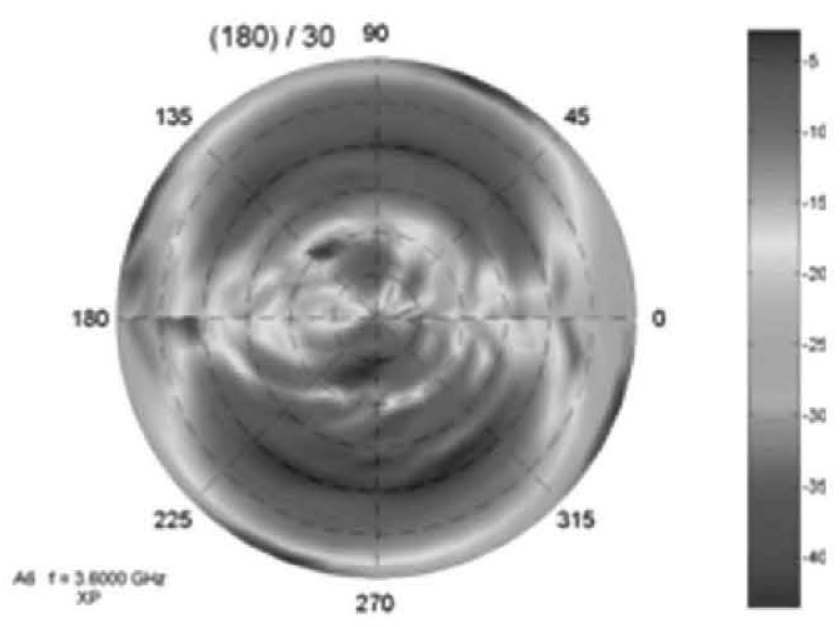

(b)

Figure 3 (a) Co-polar radiation patterns for UWB antenna at $3.6 \mathrm{GHz}$. (b) Cross-polar radiation patterns for UWB antenna at $3.6 \mathrm{GHz}$. [Color figure can be viewed in the online issue, which is available at www.interscience.wiley.com]

element are located. In Figure 1, the dimensions of the antenna are depicted, where the top view is shown with the ground plane represented in gray color, and feeding line and antenna in black.

As shown, the radiating element has a mixture between a triangular and a semicircular shape, such as a planar inverted cone antenna (PICA) [5], with a small slot inside. The dielectric supports the largest size of the structure, where $w 1=40 \mathrm{~mm}$ and $h$ diel $=55 \mathrm{~mm}$. However, the ground plane only supports the feeding line, so $h$ gnd $=35 \mathrm{~mm}$. Thus, the ground plane of the monopole is the same as the feeding line. The upper triangle has an angle of $120^{\circ}$ and a length of $L 1=10 \mathrm{~mm}$. The width of the antenna is $w 2$ and its height is given by $h 2$. On the other hand, the feeding line has been designed with a width of $1.54 \mathrm{~mm}$, and a length as same as the ground plane plus a little more space to have the monopole not being in contact with the ground plane, with $h 1$ $=2 \mathrm{~mm}$. Finally, a slot is introduced to create a resonant frequency and obtain the desired response in all frequency bands. The dimensions of the slot are $w \mathrm{~s}=3.4 \mathrm{~mm}$ and $h \mathrm{~s}=0.3 \mathrm{~mm}$.

Once the antenna has been designed by simulations in Computer Simulation Technology Mircowave Studio, the implementation and measurements are carried out. Figure 2 represents the measured reflection coefficient for the designed antenna. As it is shown, the antenna satisfies the requirement for this type of

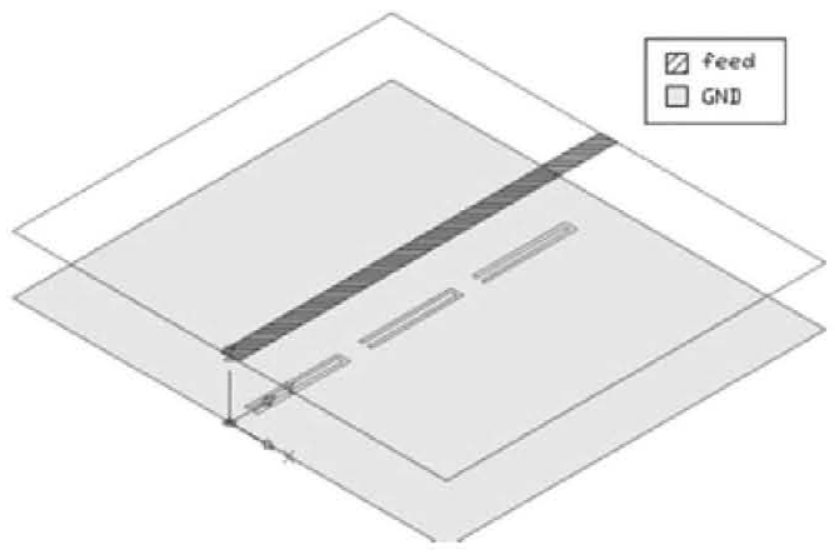

Figure 4 Representation of notch-filter design

antennas, which has to present a $\left|\mathrm{S}_{11}\right|<-10 \mathrm{~dB}$ from 3.1 to $10.6 \mathrm{GHz}$. In this case, for $5.7 \mathrm{GHz}$, the antenna has little ripples that exceed the requirements in $0.4 \mathrm{~dB}$ only.

On the other hand, the measured radiation pattern in an anechoic chamber is represented in normalized co-polar and cross-polar patterns, Figures 3(a) and 3(b), respectively, at 3.6 $\mathrm{GHz}$. The antenna gain is $2.2 \mathrm{dBi}$ and $\theta=0^{\circ}$ represents the vertical direction of Figure 1 and $\varphi=0^{\circ}$ does it for the right direction of Figure 1. The maximum power is radiated in $\varphi=0^{\circ}$ and $180^{\circ}$.

\section{NOTCH-FILTER DESIGN}

The notch filter has been designed to eliminate the $5.3 \mathrm{GHz}$ ISM band. The structure of the filter is a novel design where the key part is in the ground plane. The filter will be situated in the feeding line, so a new circuit with the dimensions of ground plane in Figure 1 has been developed. The filter uses a simple feeding line with the dimensions described in the previous section. However, the ground plane has different slots to create the filter [6]. This technique is known as photonic band gap (PBG) [7]. In this case, three U-shaped slots are introduced in the ground plane (see Fig. 4).

The notch filter has to work properly for all UWB bandwidths except for the $5.25 .4 \mathrm{GHz}$ to cancel the possible interferences. Figures 5-7 represent the study of the reflection coefficient for different parameters of U-shaped slots. Figure 5

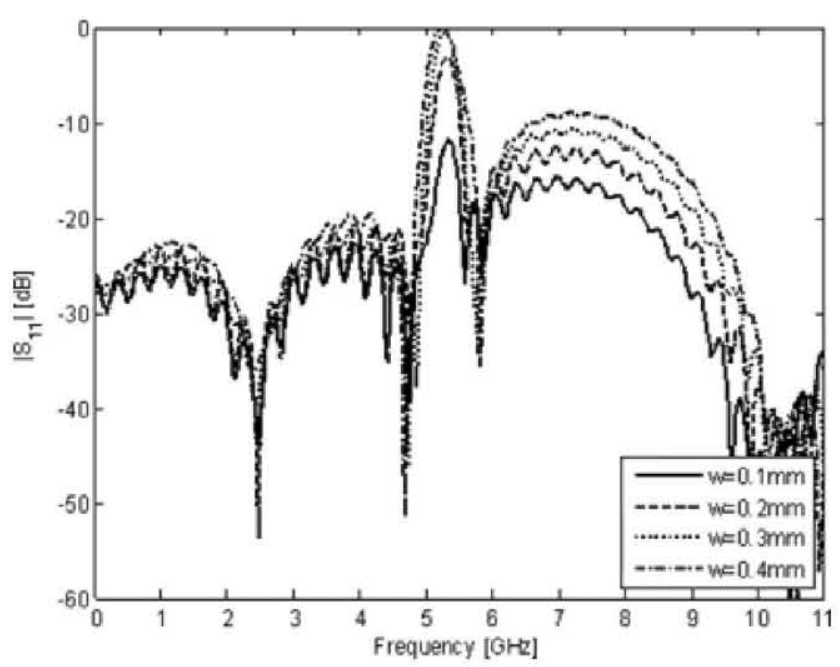

Figure 5 Reflection coefficient for different values of width of Ushaped branches 


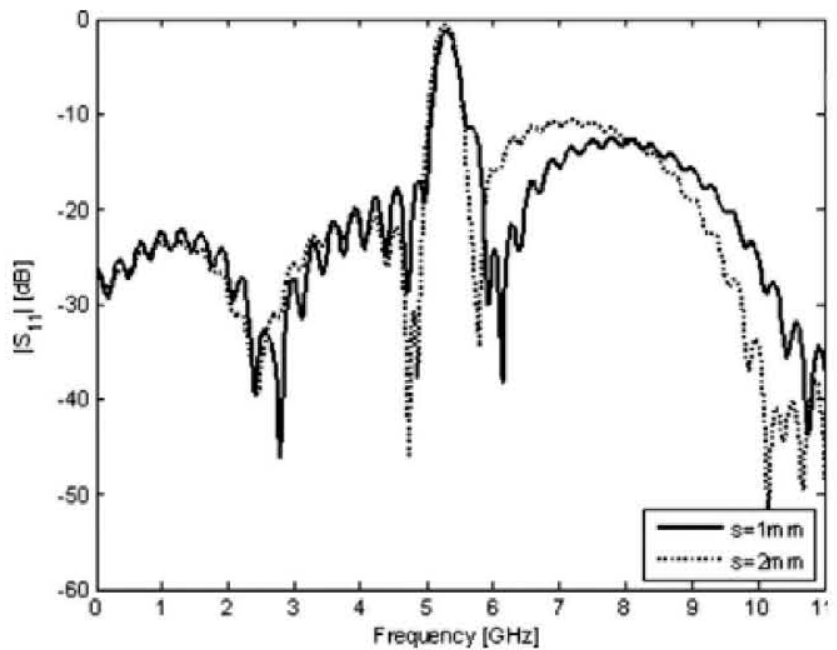

Figure 6 Reflection coefficient for different values of spacing between U-shaped slots

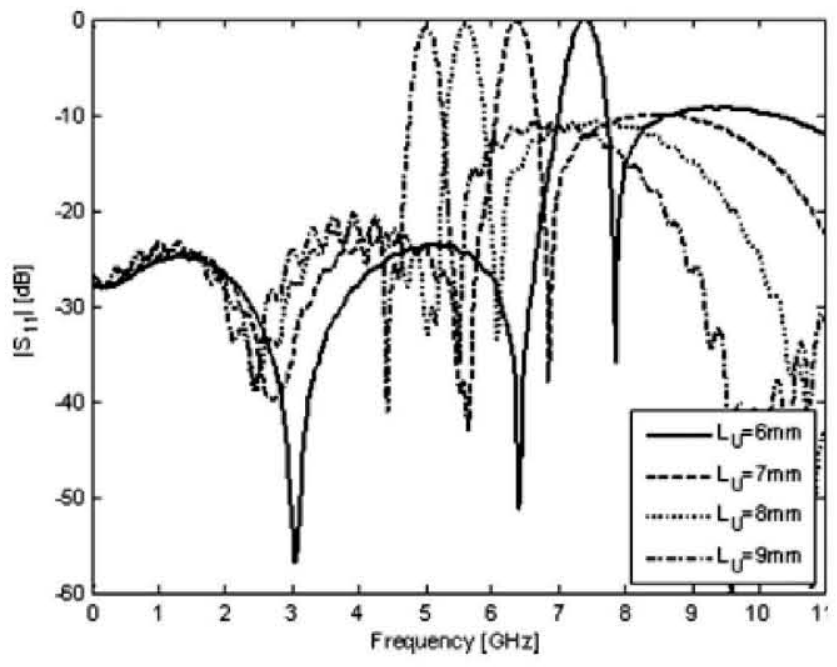

Figure 7 Reflection coefficient for different values of length of Ushaped slots

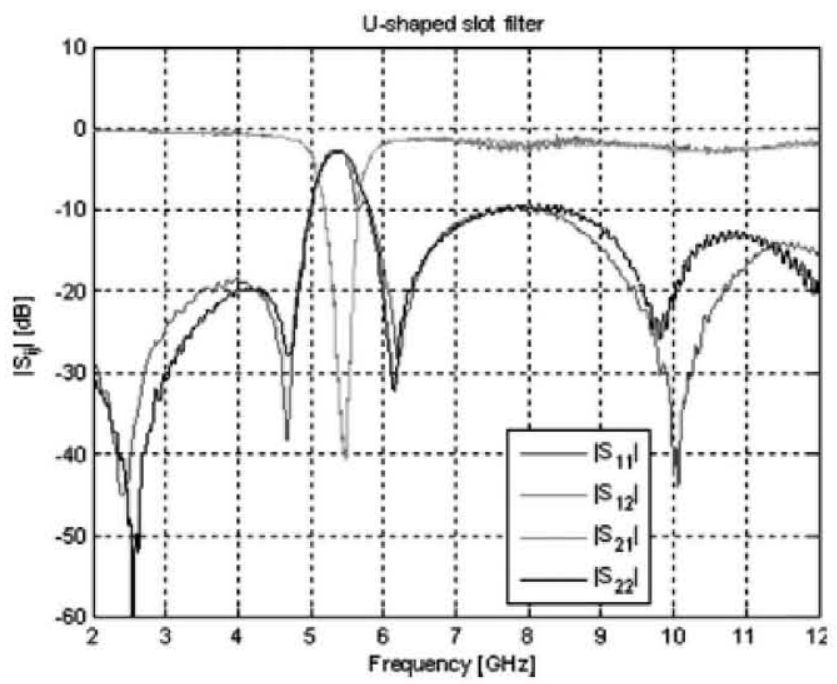

Figure 8 Scattering parameters of final notch-filter implementation. [Color figure can be viewed in the online issue, which is available at www.interscience.wiley.com]

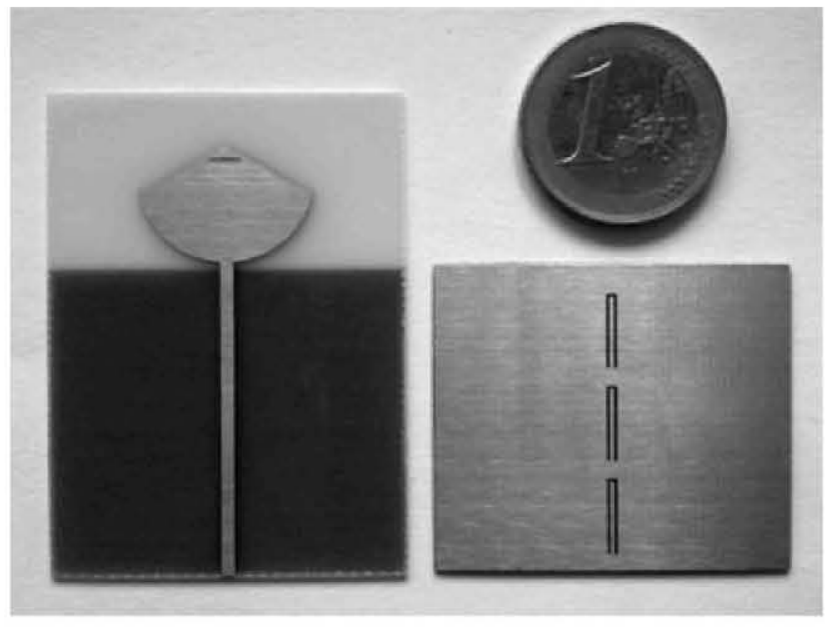

Figure 9 Final notch-filter antenna (left) and ground plane of the filter (right). [Color figure can be viewed in the online issue, which is available at www.interscience.wiley.com]

compares the reflection coefficient for different values of the width for $U$ branches. For simplicity, the U-shaped slot has the same width for the three branches. In the ISM band, the best performances are obtained for the highest value of $w$, but it has a lobe at $7.5 \mathrm{GHz}$ that is close to $-10 \mathrm{~dB}$.

On the other hand, Figure 6 represents the reflection coefficient obtained for different values of spacing between the Ushaped slots given by $s$. The results show that the filter is more selective when the spacing is higher.

And finally, the study of how the length of the slot influences the reflection coefficient is presented. The length of the slot $\left(L_{U}\right)$ was varied from 6 to $9 \mathrm{~mm}$. Figure 7 shows all the results where the notch frequency changes as $L_{U}$. Moreover, the filter selectivity grows with high values of length. Thus, this is the most important parameter to centre the filter to the desired frequency band.

Once the parametric studies were done, the following values were taken for the final design: $s=2 \mathrm{~mm}$ and $L_{U}=8 \mathrm{~mm}$.

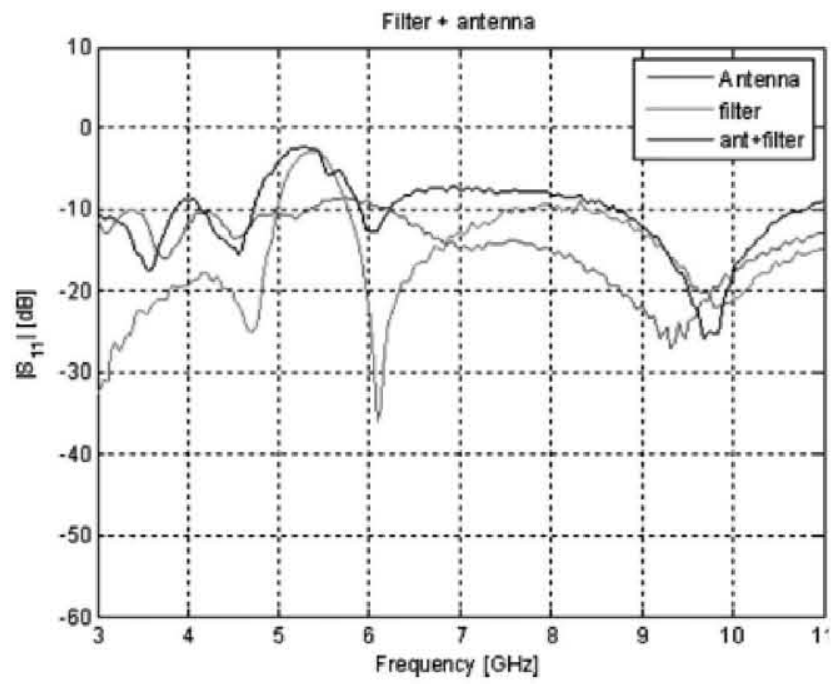

Figure 10 Reflection coefficient for the antenna, filter, and antenna notch cases. [Color figure can be viewed in the online issue, which is available at www.interscience.wiley.com] 
Figure 8 represents the measured scattering parameters of the implemented filter. The transmission coefficients for both $(1$ and 2) the ports are quite similar. However, a little variation is shown when the reflection coefficient is measured from port 1 or 2. Clearly, the results illustrate good performances for the notch filter, as there is a stop band at $5.3 \mathrm{GHz}$.

\section{NOTCH ANTENNA}

Once the antenna and the notch filter have been designed, the final implementation and the construction of the integration of different parts were realized. Figure 9 depicts the final implementation of the notch antenna, which consists of the notch filter and the antenna, i.e., a feeding line and the radiating element in the superior layer, and the ground plane with the U-shaped slots in the inferior layer. On the left, the top view of the notched filter antenna is illustrated and on the right, the ground plane for the filter case is shown. For certain UWB applications, the antenna must have a compact size, as the final designed antenna is shown in Figure 9.

Figure 10 represents the measured reflection coefficients for the cases of single antenna, single filter, and the construction of antenna with the filter. The results present good performances for the notch ISM frequency band at $5.3 \mathrm{GHz}$. Nevertheless, and due to the matching of the filter from 6.3 to $8.6 \mathrm{GHz}$, the final implementation obtains a reflection coefficient less than $-8 \mathrm{~dB}$.

\section{CONCLUSIONS}

In this paper, a novel and a compact ultra-wideband antenna has been presented. The antenna is based on microstrip structure where the radiating element is a printed monopole. It has a PICA shape with a slot inside to obtain a good matching in the entire frequency band: $3.1-0.6 \mathrm{GHz}$. However, for certain applications, the interferences for ISM band at $5.3 \mathrm{GHz}$ must be mitigated. For this purpose, a notch filter has been designed based on the PBG concept. The filter has been implemented with Ushaped slots in the ground plane. A detailed study of the influence of different parameters was carried out. Finally, the antenna and the notch filter were integrated in the same printed circuit, and the results show good performances for the notch-filter antenna in the frequency range for UWB applications.

\section{ACKNOWLEDGMENT}

The electromagnetic simulations presented in this paper have been studied using Computer Simulation Technology (CST) Microwave Studio version 2006 under a co-operation agreement between CST and Universidad Politécnica de Madrid.

\section{REFERENCES}

1. H. Schantz, The art and science of ultrawideband antennas, Artech House, Boston, MA, 2005.

2. S.H. Choi, J.K. Park, S.K. Kim, and J.Y. Park, A new ultra-wideband antenna for UWB applications, Microwave Opt Technol Lett 40 (2004), pp. 399-401.

3. Y. Kim and D.H. Kwon, CPW-fed planar ultra wideband antenna having a frequency band notch function, Electron Lett 40 (2004), pp. 403-405.

4. W.J. Lui, C.H. Cheng, Y. Cheng, and H. Zhu, Frequency notched ultra-wideband microstrip slot antenna with fractal tuning stub, Electron Lett 41 (2005), pp. 9-10.

5. S.-Y. Suh, W.L. Stutzman, and W.A. Davis, A New ultrawideband printed monopole antenna: the planar inverted cone antenna (PICA), IEEE Trans Antennas Propag 52 (2004), pp. 1361-1365.
6. S.C. Bera, R.V. Singh, V.K. Garg, N.S. Arora, and S.S. Nair, A novel PBG structure for filter application, Microwave Opt Technol Lett 48 (2006), pp. 188-190.

7. P. de Maagt, R. Gonzalo, Y.C. Vardaxoglou, and J.M. Baracco, Electromagnetic bandgap antennas and components for microwave and (sub)millimeter wave applications, IEEE Trans Antennas Propag 51 (2003), pp. 2667-2677.

(C) 2009 Wiley Periodicals, Inc.

\section{A COMPACT MICROSTRIP UWB BPF WITH NONUNIFORMLY SHORT- CIRCUITED CPW RESONATOR}

\author{
H. Chen and Y.-X. Zhang \\ School of Electronic Engineering, University of Electronic Science \\ and Technology of China, Chengdu, Sichuan 610054, China; \\ Corresponding author: hchen0730@yahoo.com.cn
}

Received 12 April 2009

ABSTRACT: A novel and compact ultra-wideband bandpass filter using hybrid microstrip/coplanar waveguide is presented. The proposed filter consists of a single coplanar waveguide with nonuniformly short-circuited resonator, which is broadside-coupled to two microstrip open-circuited stubs on the other side of a common substrate. The measured results show that the filter has a good performance, including a low insertion loss, a group delay variation of less than 0.25 ns within the passband, stop-band of up to $18.8 \mathrm{GHz}$ at high frequencies, and an out-of-band rejection level of below $-16.5 \mathrm{~dB}$ from 11.4 to $18.8 \mathrm{GHz}$. (C) 2009 Wiley Periodicals, Inc. Microwave Opt Technol Lett 52: 216 218, 2010; Published online in Wiley InterScience (www.interscience. wiley.com). DOI 10.1002/mop. 24859

Key words: (UWB); microstrip; broadside-coupled; coplanar waveguide $(C P W)$; nonuniformly; short-circuited resonator

\section{INTRODUCTION}

Since the Federal Communications Commission (FCC)'s release of the frequency band from 3.1 to $10.6 \mathrm{GHz}$ for commercial communication applications in February 2002, the ultra-wideband (UWB) radio system has been receiving great attention from both academy and industry [1]. To realize such UWB radio system, an UWB bandpass filter (BPF) is one of the key passive components. Using existing parallel coupled microstrip techniques, filters with large FBW could be designed in principle [2]. However, filters with FBW $80 \%$ have been difficult to realize because of the coupling between the resonators. The designs of coplanar waveguide (CPW) BPF have also been well documented [3]; however, large FBW have not been realized. Recently, the microstrip/CPW broadside-coupled structures have been utilized to realize desired tight couplings for designing UWB filters, where the CPW resonators used are either a halfwavelength or one full-wavelength long $[4,5]$. A compact UWB parallel coupled BPF has been proposed [6], which uses a quarter-wavelength CPW uniformly short-circuited resonator and broadside-coupled to two microstrip open-end stubs in parallel on the other side of a common substrate. However, the filter with FBW 90\% has not covered completely the UWB range from 3.1 to $10.6 \mathrm{GHz}$, and the effective range of stop-band rejection below $15 \mathrm{GHz}$.

In this letter, a new UWB band-pass filter uses mixed microstrip/CPW coupled structure, which consists of a nonuniformly short-circuited resonator on the microstrip ground plane. The proposed filter shows an excellently electrical performances, 